\title{
Dependence of Barkhausen Noise in Plastically Deformed Steel on Frequency and Nonlinearity of Magnetizing Field
}

\author{
J. PAL'A AND J. BYDŽOVSKÝ \\ Slovak University of Technology, Department of Electromagnetic Theory \\ Ilkovičova 3, 81219 Bratislava, Slovak Republic \\ The Barkhausen noise was measured in plastically deformed low-carbon \\ steel at various frequencies of the magnetizing field. Strip samples magne- \\ tized by a yoke were used for investigation. The measured amplitude distri- \\ butions and power spectra are compared with the results of the Alessandro, \\ Beatrice, Bertotti, Montorsi model of the Barkhausen noise. The measure- \\ ment results at the triangular magnetizing current and the triangular mag- \\ netic field in the sample were compared and the differences will be discussed.
}

PACS numbers: 81.40.Rs, 75.60.Ej

\section{Introduction}

The Barkhausen noise (BN) method of nondestructive testing provides good sensitivity to residual stress levels and changes in the microstructure of a magnetic material. In this work, the BN was measured in plastically deformed low-carbon steel. Plastic deformation changes the average number of individual pulses in large Barkhausen jump due to the change of the dislocation density and distribution of residual microstresses in the sample. The influence of the plastic deformation on the BN is usually investigated using the defined (triangular or sinusoidal) magnetizing current with some low frequency [1]. In this paper, we tried to investigate the influence of the magnetizing frequency on the measurement results. In addition, when the triangular current from the power amplifier is used as magnetizing current in coils of the yoke, the nonhomogeneity of magnetic circuit causes that the magnetic field in the sample is not proportional to the current, and therefore the field rate is not constant [2]. Further, the magnetizing frequency and the deformation of samples heavily influence the nonlinearity of the sample magnetization characteristic, so the samples are not measured with the same field amplitude. To impose the constant field rate with the defined field amplitude, the field was measured by the tangential Hall probe on the sample surface and the digital feedback was used. The impact of this feedback on measurement results will be discussed. 


\section{Experimental}

Commercial low-carbon steel with composition $(\mathrm{C}=0.03, \mathrm{Mn}=0.18$, $\mathrm{Si}=0.13, \mathrm{P}=0.027, \mathrm{~S}=0.027, \mathrm{~N}=0.007 \mathrm{wt} . \%)$ was chosen for the investigation. The strip samples were tested after unloading by a single yoke method under full PC control [3]. The driving coils were wound directly on the yoke legs of the same material as the sample. The sample field $H$ was evaluated either directly from the magnetizing current (measurement with the defined magnetizing current of the amplitude of $1 \mathrm{~A}$ ) or from the magnetic field measured at the sample surface using the Hall probe (measurement with the defined magnetic field of the amplitude of $2.5 \mathrm{kA} / \mathrm{m}$ ). The BN was measured by a perpendicular sensing coil.

\section{Results and discussion}

The dependences of the amplitude distribution and the power spectrum on the plastic deformation for the magnetizing frequency of $0.25 \mathrm{~Hz}$ are shown in Fig. 1. The measured amplitude distributions and power spectra (points in Fig. 1) were fitted with the results of the Alessandro, Beatrice, Bertotti, Montorsi (ABBM) model [4] (lines in Fig. 1): the amplitude distribution of the form $P(u)=k_{u} u^{-1} \exp \left(-k_{\mathrm{e}} u\right)$ and the power spectrum in high frequency region of the form $S(f)=k_{\mathrm{s}} / f^{2}$, where $k_{u}, k_{\mathrm{e}}$ and $k_{\mathrm{s}}$ are fitting constants, $u$ is the Barkhausen voltage, and $f$ is the frequency. From the figure we can see that the amplitude distribution decreases with the deformation at high levels of the voltage and the power spectrum decreases at all levels of the frequency, mainly due to the creation of dislocations in the deformed material [3].
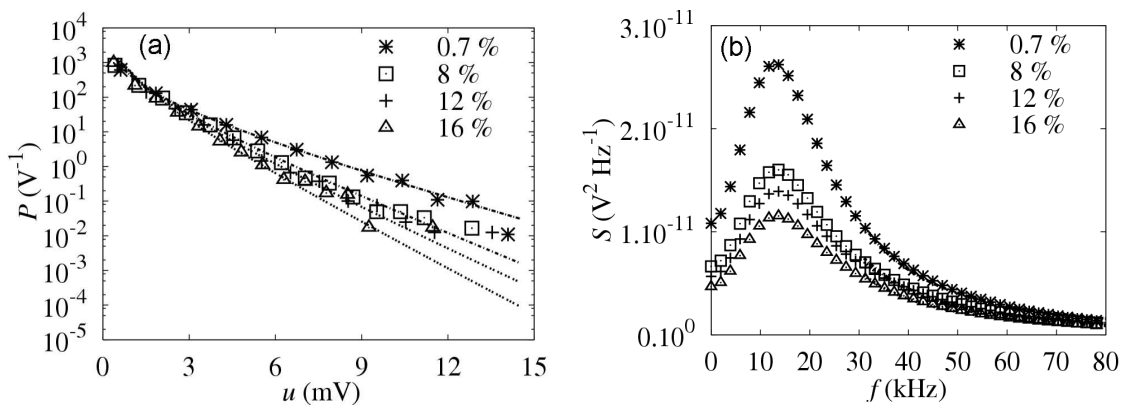

Fig. 1. Dependences of the amplitude distribution (a) and the power spectrum (b) on the strain for the triangular magnetizing current.

The BN changes also with the magnetizing frequency because of overlapping the clustered independent jumps at different positions in the sample [1]. The increasing magnetizing frequency at constant maximum field intensity causes the increase in the number of jumps per unit time and therefore raising influence of overlapping. The overlapping is supposed to be supported by raising the number of domain walls and therefore increasing the number of interactions between the 
domain walls and pinning sites [5]. Therefore the root mean square (RMS) value of the $\mathrm{BN}$ increases with the magnetizing frequency and the number of Barkhausen jumps decreases above $0.125 \mathrm{~Hz}$ (Fig. 2b). The drop of the number of jumps below $0.125 \mathrm{~Hz}$ is caused by decreasing the magnitude of some jumps below a measurable value. The increase in the sensitivity of the RMS value on deformation with decreasing magnetizing frequency (Fig. 2a) can then be attributed to the increase in the number of jumps and consequent relative raise of the BN levels, which are influenced by the deformation.
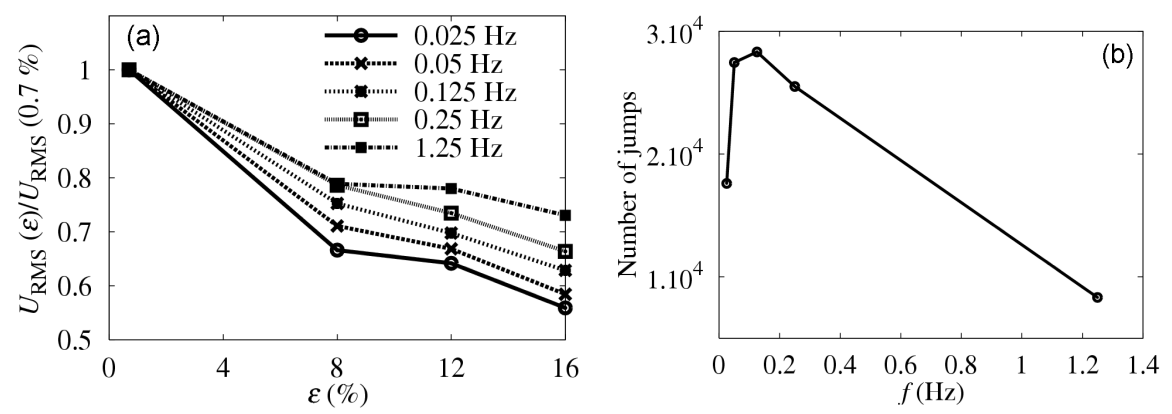

Fig. 2. Normalized dependences of the RMS value of the BN on the strain $\epsilon$ for different magnetizing frequencies (a) and dependence of the number of the Barkhausen jumps on the magnetizing frequency $f$ for $8 \%$ of strain, measured at the triangular magnetizing current (b).
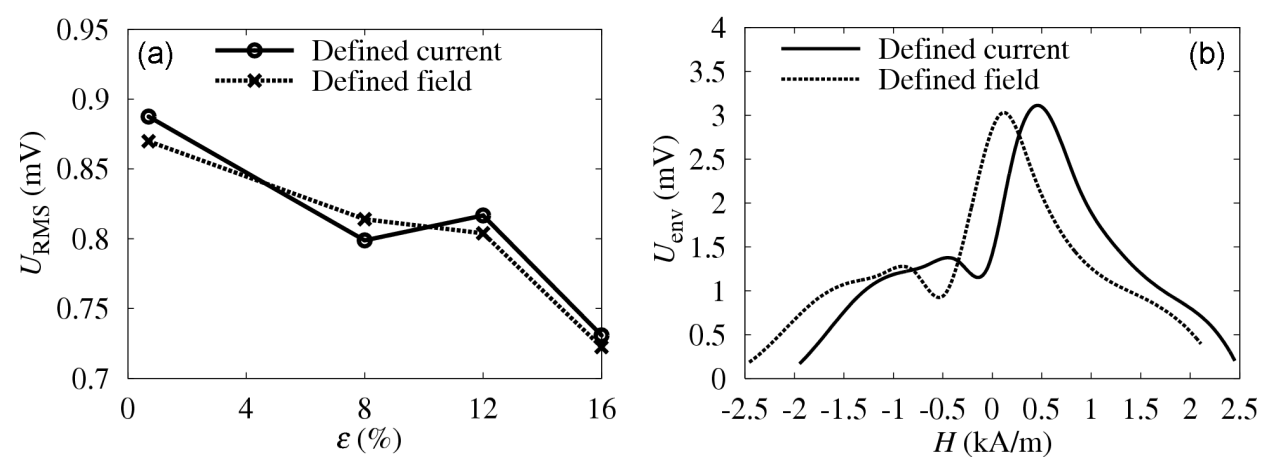

Fig. 3. RMS values (a) and envelopes (b) of the BN for defined triangular magnetizing current, respectively defined triangular magnetic field with frequency of $1 \mathrm{~Hz}$.

The comparison of the RMS values and the envelopes for the defined magnetizing current, respectively defined magnetic field is shown in Fig. 3. The amplitude of the sample field was chosen such that the magnetizing current in the case of the $0.7 \%$ deformed sample was equal to the amplitude of the magnetizing current in the mode with the defined magnetizing current. Measuring with the 
constant amplitude of the sample field should cause the difference in amplitudes of the magnetizing current of different samples. However, this difference has only slight effect on the RMS value of the BN (Fig. 3a). The nonlinear change of the sample field with the time in the case of the defined magnetizing current causes that the position of the envelope peak calculated from the magnetizing current is significantly moved from the position obtained using the Hall probe (Fig. 3b).

\section{Conclusions}

Optimizing the frequency of the magnetizing process can increase the sensitivity of the BN on the plastic deformation. Even though using the defined magnetic field in the sample changes the RMS value of the BN only slightly in comparison with the case of the defined magnetizing current, the position of the envelope peak moved significantly.

\section{Acknowledgments}

This work was supported by VEGA Grant No. 1/3116/06.

\section{References}

[1] D.G. Hwang, H.C. Kim, J. Phys. D, Appl. Phys. 21, 1807 (1988).

[2] J. Pal'a, O. Stupakov, in: Elitech, Conf. Proc., Ed. P. Ballo, Faculty of Electrical Engineering and Information Technology, Slovak University of Technology, Bratislava 2005, p. 114.

[3] O. Stupakov, J. Pal'a, I. Tomáš, J. Bydžovský, V. Novák, Mater. Sci. Eng. A 462, 351 (2007).

[4] B. Alessandro, C. Beatrice, G. Bertotti, A. Montorsi, J. Appl. Phys. 68, 2901 (1990).

[5] A. Dhar, D.L. Atherton, IEEE Trans. Magn. 28, 3363 (1992). 